Document downloaded from:

http://hdl.handle.net/10251/65826

This paper must be cited as:

Hervás Peralta, J.; Fernandez-Pousa, C.; Barrera Vilar, D.; Pastor Abellán, D.; Sales Maicas, S.; Capmany Francoy, J. (2015). An Interrogation Technique of FBG Cascade Sensors Using Wavelength to Radio-Frequency Delay Mapping. Journal of Lightwave Technology. 33(11):2222-2227. doi:10.1109/JLT.2015.2409318.

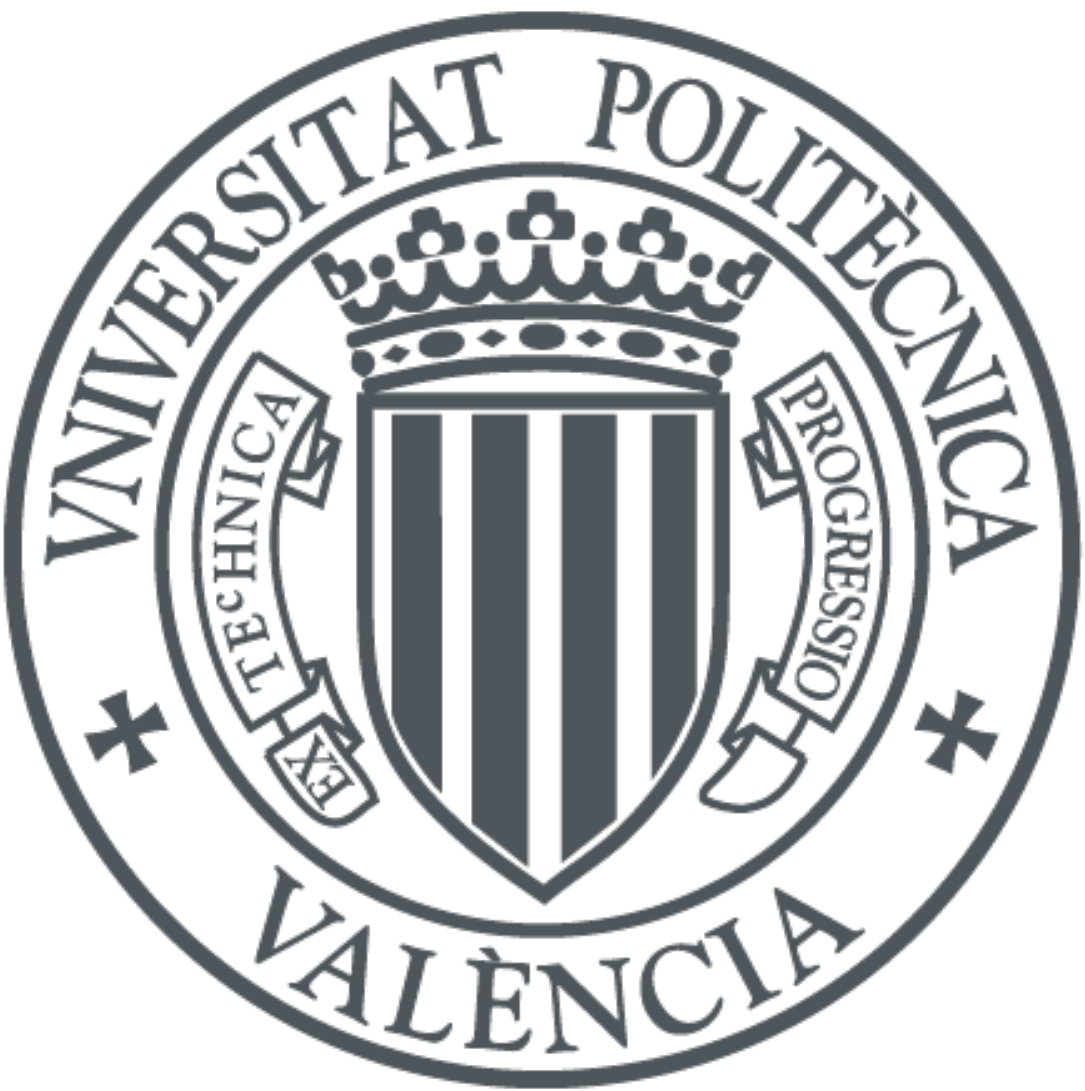

The final publication is available at

http://dx.doi.org/10.1109/JLT.2015.2409318

Copyright Institute of Electrical and Electronics Engineers (IEEE)

Additional Information 


\title{
An interrogation technique of FBG cascade sensors using wavelength to radio-frequency delay mapping
}

\author{
Javier Hervás, Carlos R. Fernández-Pousa, Senior Member, OSA, IEEE, David Barrera, Daniel Pastor, \\ Salvador Sales, Senior Member, IEEE and José Capmany, Fellow, OSA, IEEE
}

\begin{abstract}
A technique is proposed to interrogate a distributed photonic sensor composed of seven standard fiber Bragg gratings in cascade. A microwave-modulated optical signal is launched into a FBG cascade and subsequently undergoes dispersion in standard single-mode fiber, so that the different Bragg wavelengths are mapped into the radio-frequency (RF) delays that determine the peaks in the electrical impulse response of the optical link. Shifts in Bragg wavelengths have been determined from the shifts in the impulse response's peaks by recording the link's $\mathrm{RF}$ response in the range dc-20 $\mathrm{GHz}$ using a Vector Network Analyzer (VNA), and then performing its inverse Fourier transform. A resolution of $14 \mathrm{pm}$ is experimentally demonstrated after zero-padding the VNA traces.
\end{abstract}

Index Terms-Optical fiber sensors, Bragg gratings, Microwave Photonics, analog optical links.

\section{INTRODUCTION}

Optical fiber sensors continue to attract attention due to not only the low loss, lightweight and immunity to electromagnetic interference of the optical fiber [1], but also to the possibility of implementing distributed [2,3,4] and multiplexed sensing. In multiplexed sensors, also known as quasi-distributed sensors, a number of discrete sensors, such as interferometers [5] or fiber Bragg gratings (FBGs) [6], are cascaded along a single fiber. Both optical time-domain (OTDR) [7] and frequency-domain reflectometry (OFDR) [8] can be used to interrogate these serial discrete sensing elements. In OTDR, an optical pulse is launched into the sensor to resolve spatially the reflectivity along the fiber as a function of the arrival time. This procedure thus relies on the determination of the system's electrical impulse response. The

Manuscript received xxxxxxxx x, 2014; revised xxxxxxxx x, 2014; accepted xxxxxxxxx x, 2014. Date of publication xxxxxxxxxx x, 2014; date of current version $\mathrm{xxxxxxxx} \mathrm{x}, 2014$. This work was supported in part by the Spanish MINECO through projects TEC2014-60378-C2-1-R and -2-R, by the EU Infraestructura FEDER operative program 2007-2013, and by the Government of Valencia through the Research Excellency Award Program GVA PROMETEO 2013/012. J. Hervás work is supported by the MECD FPU scholarship (FPU13/04675)

J. Hervás, D. Barrera, D. Pastor, S. Sales, and J. Capmany are with the iTEAM Research Institute, Optical Quantum and Communications Group, Universidad Politécnica de Valencia, Valencia 46022, Spain (e-mail: jaherpe2@teleco.upv.es; dabarvi@iteam.upv.es; dpastor@dcom.upv.es; ssales@dcom.upv.es; jcapmany@iteam.upv.es).

C. R. Fernández-Pousa is with the Departamento de Ingeniería de Comunicaciones, Universidad Miguel Hernández, Elche 03202, Spain (e-mail: c.pousa@umh.es).

Color versions of one or more of the figures in this paper are available online at http://ieeexplore.ieee.org

Digital Object Identifier xxxxxxxxxxxxxxxxxxxxx
OFDR techniques, in turn, can be grouped as coherent or incoherent [8]. Incoherent OFDR is based on the capture of the system's electrical frequency response and subsequent computation of the electrical impulse response by inverse Fourier transformation (IFT), whereas in coherent OFDR the reflectrometric trace is obtained as the IFT of an optical interference signal between reference and reflector when scanned by swept laser source.

On the other hand, FBGs are widely used as point or quasidistributed sensors due to its versatile and mature fabrication technology. Long, high-reflectivity FBGs have been used to detect hot spots [6], and a temperature sensor based on a 10cm long continuous weak FBG has been reported [4]. In addition, in [3] it was presented a multiplexed sensor based on cascading weak FBGs, a scheme that does not require the difficult fabrication of long, continuous weak FBGs.

In this paper, we use a multiplexed FBG sensor composed of seven FBGs written in cascade where, in contrast to [3], the gratings are standard, not weak. The Bragg wavelength shift under environmental changes constitutes the sensing principle, and the proposed interrogation method can be viewed as a modification of the incoherent OFDR technique. The FBG peak reflection wavelengths are mapped into radio-frequency (RF) delays by use of a dispersive delay line, here implemented with a single-mode fiber line, and these delays determined from the IFT of the system's electrical frequency response. To illustrate the flexibility of the proposed concept further, neither the differences between the Bragg wavelengths of adjacent FBGs in the cascade, nor their shapes, widths, or reflected power, are uniform. As will be shown in this paper, even in this general scenario the wavelength to radiofrequency delay mapping technique together with numerical post processing of the retrieved frequency-domain traces allow for an effective interrogation of the multiplexed FBG sensor.

\section{OPERATION PRINCIPLE}

The setup used to interrogate the sensor, considered here as the device under test (DUT), is depicted in Fig. 1. It is based on the measurement of the electrical frequency response $H(\omega)$, or scattering parameter $S_{21}(\omega)$, relating the output and input RF signals, as measured by a vector network analyzer (VNA), when the DUT is considered as an optical transmission system. 


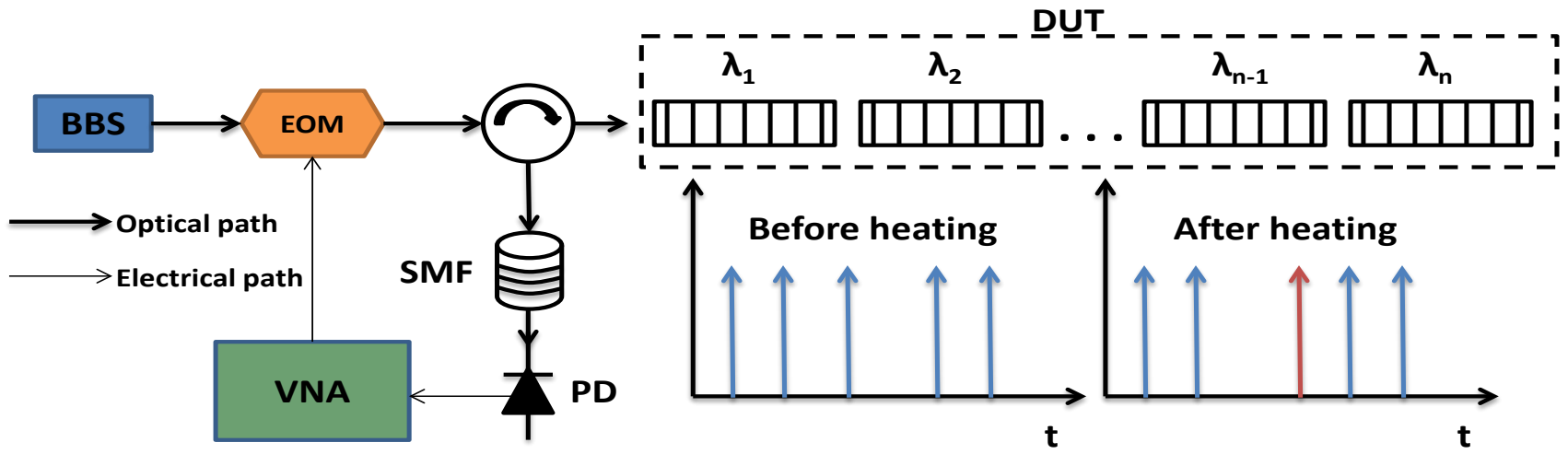

Fig. 1. Schematic diagram of the setup used to measure the system's electrical frequency response and principle of operation. After heating or stretching one of the FBGs, its Bragg wavelength shifts. This wavelength shift is transformed by the dispersive delay line into a time delay shift in the corresponding peak of the electrical impulse response, as shown with a red arrow.

The output power of a C-band broadband optical source (BBS), implemented here by use of a SLED, is modulated by an electro-optic modulator (EOM) by the RF signal from the VNA output port. This optical signal is then launched into the DUT by means of a circulator. In the DUT, different spectral slices of the modulated BBS are reflected by the FBGs and routed to a dispersive element composed of $6.83 \mathrm{~km}$ of single mode fiber (SMF). This dispersive element is used to map the Bragg wavelengths of the individual FBGs into electrical delays of the RF modulating waveforms, so that shifts in Bragg wavelength due to temperature or strain induce group delay shifts. After photodetection (PD) the RF signal is directed to the VNA input port and the electrical response of the system is measured as function of the input RF tone, $\omega$. The electrical response has the form [3]:

$$
H(\omega)=\sum_{k=0}^{N-1} a_{k} e^{-j \omega \tau_{k}}
$$

where $N$ is the number of FBGs or samples, $a_{k}>0$ is the sample weight, proportional to the FBG reflectivity and thus positive, and $\tau_{k}$ is the sample delay, which is given by the group delay $\tau(\lambda)$ experienced by the $k$-th Bragg wavelength, $\tau_{k}$ $=\tau\left(\lambda_{B k}\right)$, in the SMF dispersive delay line. The frequency response (1) is thus the superposition of $N$ oscillations in frequency, and the sample delays, which determine the corresponding Bragg wavelengths, can be extracted by an IFT.

VNA traces provide a discrete version of the amplitude and phase of function $H(\omega)$ in a range of positive frequencies Denoting the scanning frequency interval as $0<\omega<2 \pi B$, with $B$ the measurement bandwidth in Hertz, the IFT of (1) is:

$$
h_{B}(t)=B \sum_{k=0}^{N-1} a_{k} \mathrm{e}^{j \pi B\left(t-\tau_{k}\right)} \operatorname{sinc}\left[B\left(t-\tau_{k}\right)\right]
$$

where $\operatorname{sinc}(x)=\sin (\pi x) /(\pi x)$ and the delays can be determined from the $N$ peak positions of the sinc pulses in the amplitude of the impulse response. The peaks or pulses are assumed isolated from each other so that their overlap is negligible. We also point out that (2) is a complex impulse response obtained by IFT from the raw VNA traces in the single-sided, positivefrequency range. As a response in the electrical domain that compares input and output voltage waveforms, the link's true electrical impulse response is a real quantity, which can be retrieved from VNA data through IFT after the extension to negative frequencies by use of the hermiticity condition $H(-\omega)$ $=H(\omega)^{*}$. However, this extension would require doubling the initial data string with the subsequent numerical effort, and is not necessary in the present method since the delays can be determined directly from (2).

From $\tau_{k}=\tau\left(\lambda_{B k}\right)$, a temperature or strain measurement method can be built by comparing the delays $\tau_{k}^{0}=\tau\left(\lambda_{B k}^{0}\right)$ when the DUT is in a reference position, free of strain and at a given temperature, with the impulse response when one or several FBGs undergo a shift in Bragg wavelength due to a temperature or strain change, $\tau_{k}=\tau\left(\lambda_{B k}^{0}+\Delta \lambda_{B k}\right)$. Delay shifts $\Delta \tau_{k}=\tau_{k}-\tau_{k}^{0}$ are related to wavelength shifts according to:

$$
\frac{\Delta \tau_{k}}{\Delta \lambda_{B k}}=\left.\frac{d \tau}{d \lambda}\right|_{\lambda=\lambda_{B k}}=D\left(\lambda_{B k}\right) L
$$

where $D(\lambda)$ is the fiber's dispersion at wavelength $\lambda$ and $L$ the fiber length. Then, only the FBG temperature/strain coefficient is needed to turn this Bragg wavelength shift into a temperature or strain shift.

In (3) we have neglected second-order dispersion effects in the delay shifts induced by wavelength shifts. In our $6.83-\mathrm{km}$ SMF coil, typical shifts of $\Delta \lambda_{B k} \sim 1 \mathrm{~nm}$ induce variations $\left|D\left(\lambda_{B k}+\Delta \lambda_{B k}\right)-D\left(\lambda_{B k}\right)\right| L=S\left(\lambda_{B k}\right) L<0.4 \mathrm{ps} / \mathrm{nm}$ with respect to $D\left(\lambda_{B k}\right) L \sim 116 \mathrm{ps} / \mathrm{nm}$, which implies a relative accuracy of $0.4 / 116 \sim 4 \times 10^{-3}$ in the determination of $\Delta \lambda_{B k}$. For $\Delta \lambda_{B k} \sim 1$ $\mathrm{nm}$, higher-order dispersion corrections to (3) are to be taken into account for target resolutions $\leq 4 \mathrm{pm}$, otherwise they can be neglected as is the case in our experiments.

When the IFT is calculated from discrete data $H\left[\omega_{n}\right]$, it is known [9] that the difference $\Delta t=t_{n+1}-t_{n}$ between sampling instants in the retrieved impulse response $h_{B}\left[t_{n}\right]$ is given by the inverse of the frequency span of the initial data, $H\left[\omega_{n}\right]$. If the single-sided raw data from the VNA are used in the IFT, then $\Delta t=1 / B$. However, this procedure cannot determine accurately the position of the delays in $h_{B}(t)$ since, as given by (2), they are embedded in a sinc peak of width between zeroes equal to $2 / B$. The standard numerical method to interpolate the discrete $h_{B}\left[t_{n}\right]$ trace in order to clearly resolve the peaks, consists in numerically enlarging the bandwidth to $B^{\prime}>B$ by adding zeroes to the initial data $H\left[\omega_{n}\right]$, a procedure that is referred to as zero padding. The difference between sampling points in the trace 
$h_{B}\left[t_{n}^{\prime}\right]$ so obtained is reduced by the ratio of bandwidths $F=$ $B^{\prime} / B$, i.e., $\Delta t^{\prime}=1 / B^{\prime}=\Delta t / F$.
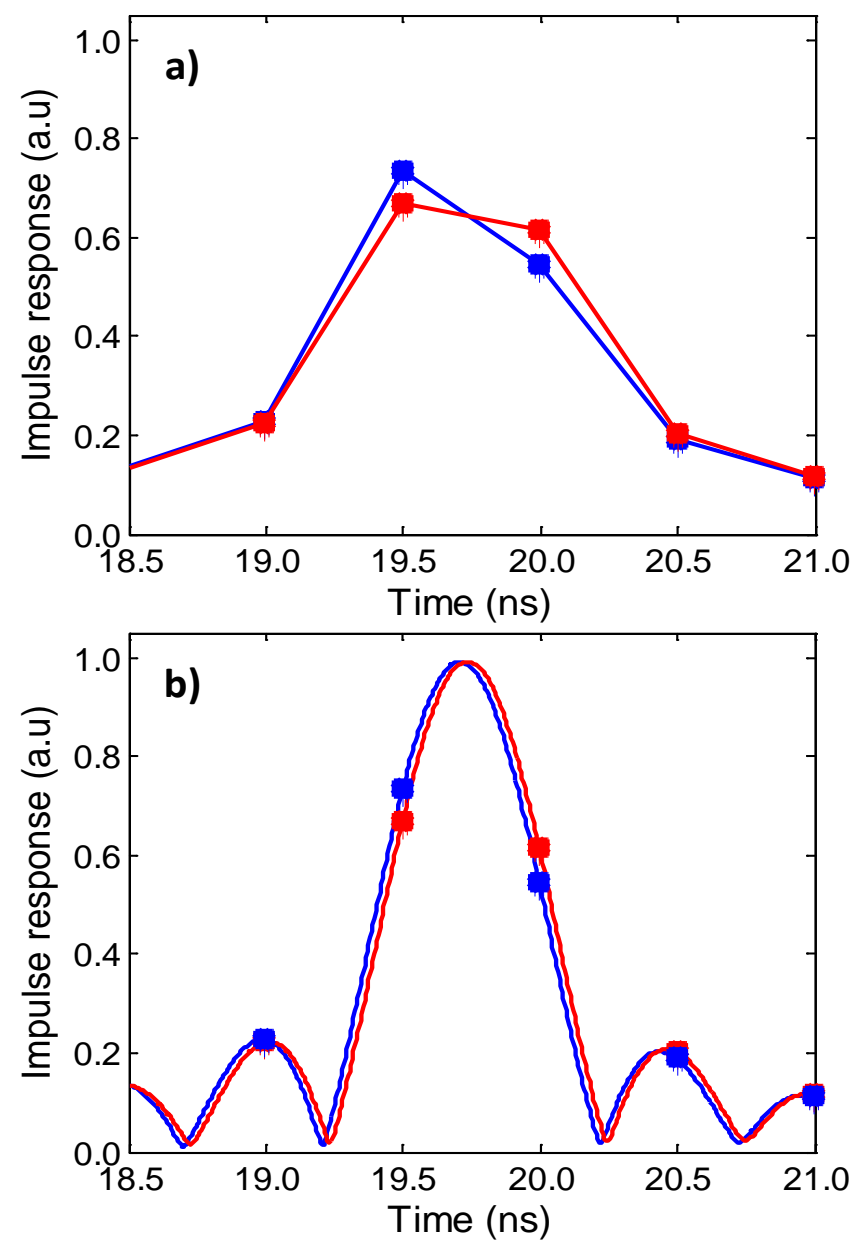

Fig. 2. Simulation of two impulse response measurements in a FBG. Blue traces: reference position, red traces: after variation of the Bragg wavelength. a): without zero padding. b): with zero padding. The dots are the values without zero padding (same as on a)), plotted for comparison.

As is well-known in digital signal processing [9], if zero padding is used to estimate the Fourier transform of a number of sinusoids such as (1), the resolution is ascribed to the width of the smallest spectral line, i.e., to the width $2 / B$ of the sinc peak in (2). In this regard, it is clear that zero padding does not increase resolution, just interpolates the Fourier transform. In our case, however, the sensor resolution is ascribed to the ability to determine the peak positions in time and is given by $\Delta t^{\prime}=1 /\left(2 B^{\prime}\right)$. Therefore, zero padding allows for an increase of resolution without an increase in measurement bandwidth. Conversely, zero padding can be used to reduce the measurement bandwidth $B$ for a given resolution $\Delta t$, by compensating the decrease in bandwidth with a higher factor F.

In Fig. 2 we present an example of the proposed procedure, where simulated data are used to determine the delay $\tau_{k}$ in the impulse response associated to a single FBG when the reflector is in a reference position (blue trace) or heated (red), resulting in a small variation of the peak's center. In the left hand side, simulated data (10001 points) in a bandwidth $B=2$ $\mathrm{GHz}$ result in temporal traces with sampling step $\Delta t=1 / B=$
$0.5 \mathrm{~ns}$, unable to resolve the temperature change. By contrast, in the right side the data trace was zero-padded to one million points, thus increasing the effective bandwidth by a factor $F=100$ up to $200 \mathrm{GHz}$. After IFT, the impulse response trace is sampled at $\Delta t^{\prime}=5 \mathrm{ps}$, revealing the structure of the sinc pulse. In this situation, the temperature change can be clearly resolved.

\section{EXPERIMENTAL MEASUREMENTS AND RESULTS}

As mentioned above, the DUT consisted on seven FBGs at different Bragg wavelengths. The widths and shapes of the reflected spectrum were also different, as shown in Fig. 3. The variations in reflected power are caused by the use of a SLED with non-uniform spectral power. Electrical measurements were performed following the scheme in Fig. 1 in a bandwidth $B=20 \mathrm{GHz}$. Due to the small length $(6.83 \mathrm{~km})$ of SMF no carrier suppression effects were observed within this frequency span. In any case, carrier suppression amounts to low-pass filtering the electrical response (1), and thus induce an additional widening of the peaks in the impulse response (2), but does not change their positions. The proposed method is expected to tolerate situations with moderate carrier suppression, or other low-pass filtering effects such as moderate limitations in modulation or detection bandwidths.

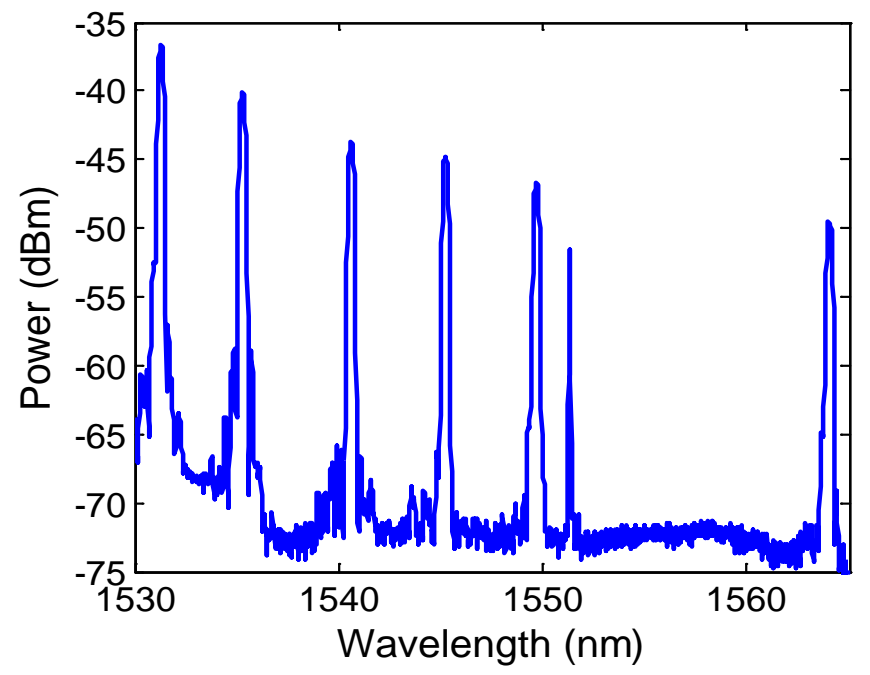

Fig. 3. Reflected spectrum of the seven FBGs.

The measurements were performed by scanning the system's 20-GHz electrical response with 32000 data points at an intermediate-frequency bandwidth of $1 \mathrm{kHz}$. The scanning or acquisition time is $\sim 20 \mathrm{~s}$. At the same time, the optical spectrum reflected by the FBGs was monitored in an optical spectrum analyzer (OSA) in order to have a comparative measurement of the shifts in Bragg wavelengths, which were induced by heating the DUT at different positions. In Fig. 4 we show the shifts observed in the OSA when the FBG at $1540.6 \mathrm{~nm}$ was heated. The reference FBG position is determined by a Bragg wavelength of $1540.6067 \mathrm{~nm}$ at room temperature, which shifts by $0.5711 \mathrm{~nm}$ to a final Bragg wavelength of $1541.1778 \mathrm{~nm}$.

The system's electrical frequency response was captured simultaneously. A zoom view of the power response at $\sim 6$ $\mathrm{GHz}$ is shown in Fig. 5, where the traces show a typical wavy 
form due to the composition of seven oscillations. Afterwards, the impulse responses were computed before and after heating by use of the IFT once zero-padding the raw traces to one million points. This gives an effective bandwidth $B^{\prime}=625$ $\mathrm{GHz}$ or a temporal resolution in the impulse response $\Delta t^{\prime}=1.6$ ps. From (3), and using a typical value of dispersion $D=17$ $\mathrm{ps} / \mathrm{nm} \cdot \mathrm{km}$, the system's wavelength shift resolution in these experiments is $\Delta \lambda_{B}=14 \mathrm{pm}$.

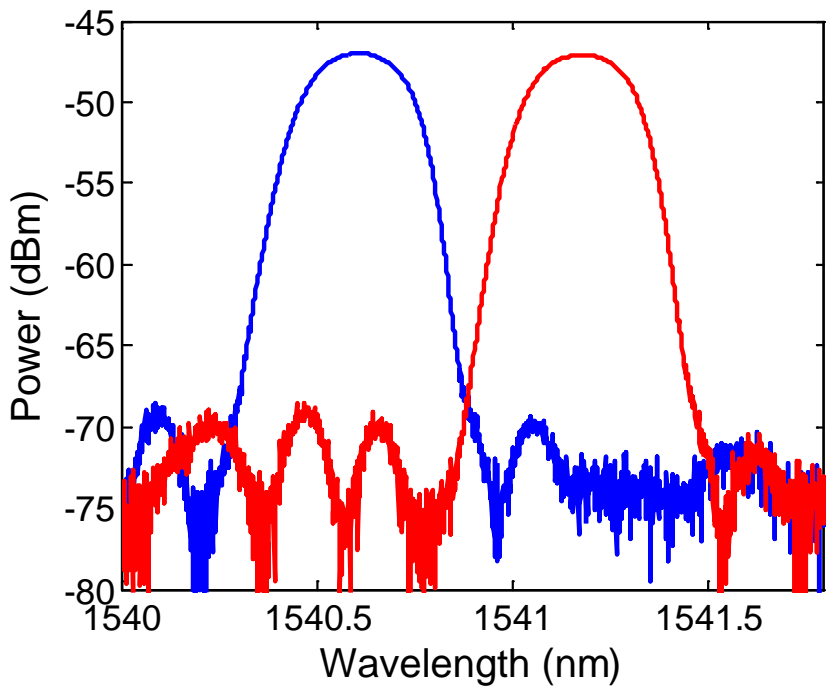

Fig. 4. Bragg wavelength shift in the FBG at $1540.6 \mathrm{~nm}$ measured with the OSA. Blue trace: reference position; red trace: FBG heated.

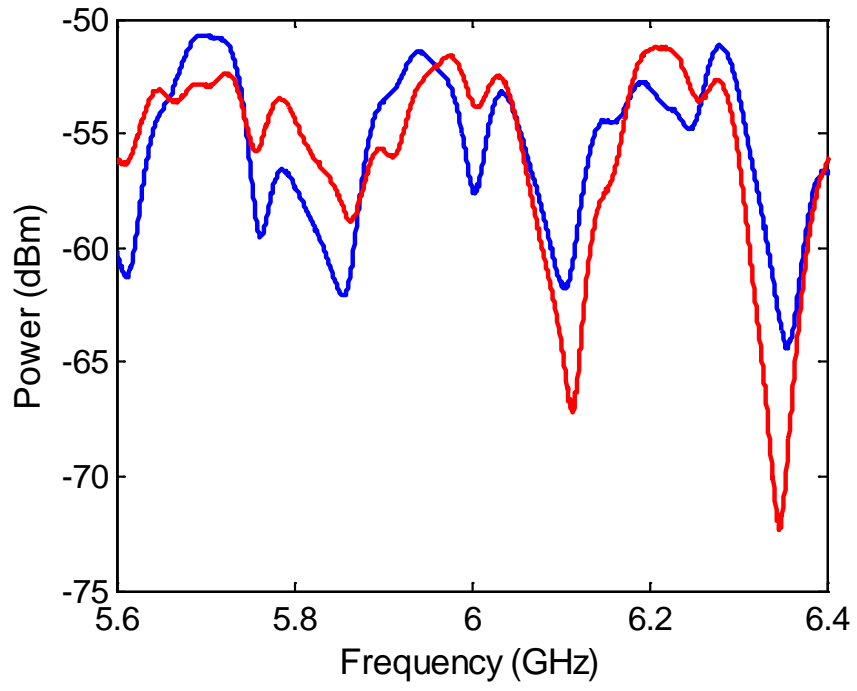

Fig. 5. Zoom view of the spectrum of the system when the DUT is under a temperature shift (red trace) and when it is not (blue trace).

The impulse response, centered at the peak generated by the heated FBG, is shown in Fig. 6. The time axis does not correspond to the true delays experienced by the microwave signal in the SMF line since it is aliased due to the large frequency step used in the VNA measurements $(\Delta f=20 \mathrm{GHz}$ / $32000=625 \mathrm{kHz}$ ). Since the present method is based on relative time measurements, this fact does not affect its performance.

It can be observed that the impulse response's peaks corresponding to the two situations arrive at different times: the blue trace (not heated) has its maximum at $479.202 \mathrm{~ns}$, whereas the maximum of the red trace (heated) is at 479.267 ns. After (3), and using a measured dispersion at the Bragg wavelength equal to $16.9036 \mathrm{ps} / \mathrm{nm} \cdot \mathrm{km}$, we obtain a Bragg wavelength shift of $0.5631 \mathrm{~nm}$. The difference with the OSA measurement is as low as $8.0 \mathrm{pm}$, under the expected resolution of the present implementation of the method.

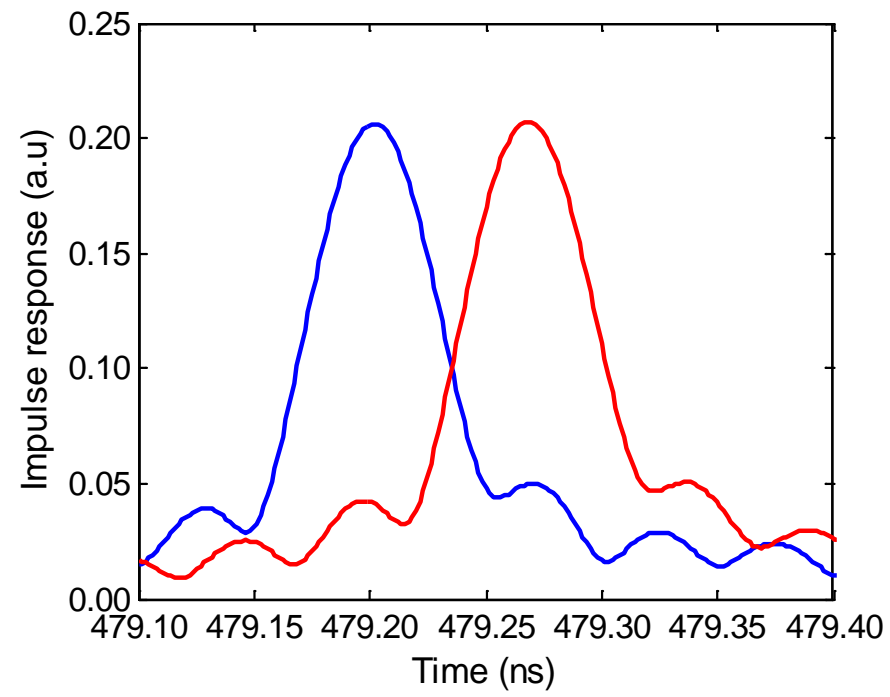

Fig. 6. Peak in the system's impulse response generated by the FBG at 1540.6 $\mathrm{nm}$ at the reference position (blue trace) and when it is heated (red trace).

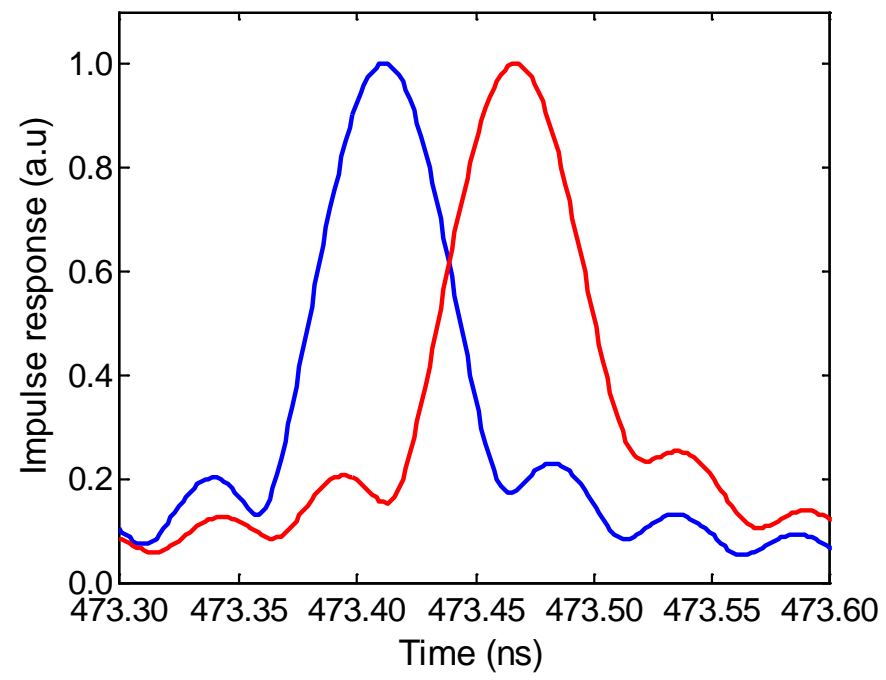

Fig. 7. Peaks in the system's impulse response generated by the FBG at $1531.3 \mathrm{~nm}$ at the reference position (blue trace) and when it is heated (red trace).

Different FBGs were heated in order to show that their shape or reflected power have no influence in the interrogation method. The results of one of these measurements, performed with the same set of parameters, has been depicted in Fig. 7 . The measured displacement in Bragg wavelength from the OSA was $0.5021 \mathrm{~nm}$, whereas the outcome of the analysis of the impulse response provides a shift of is $0.4972 \mathrm{~nm}$, using $D$ $=16.5067 \mathrm{ps} / \mathrm{nm} \cdot \mathrm{km}$ at the Bragg wavelength. The difference of $4.9 \mathrm{pm}$ is again under the expected resolution. 
Finally, we show that zero padding allows for reducing the measurement bandwidth $B$ without degradation in resolution. To show this, we low-pass filtered the 32000 points of the raw VNA data for the initial bandwidth $B=20 \mathrm{GHz}$ to the first 8000 points lying in the range below $5 \mathrm{GHz}$. After truncation, we add zeros after the data points before the IFT computation in two different configurations. The resulting string lengths were 100000 (effective bandwidth $B^{\prime}=62.5 \mathrm{GHz}$ ) and one million $\left(B^{\prime}=625 \mathrm{GHz}\right)$. In the first case, we get impulse responses from $5-\mathrm{GHz}$ data with lower resolution $\left(\Delta t^{\prime}=16 \mathrm{ps}\right)$, whereas in the second case we have an equivalent as before $\left(\Delta t^{\prime}=1.6\right.$ ps). The four traces are shown in Fig. 8.

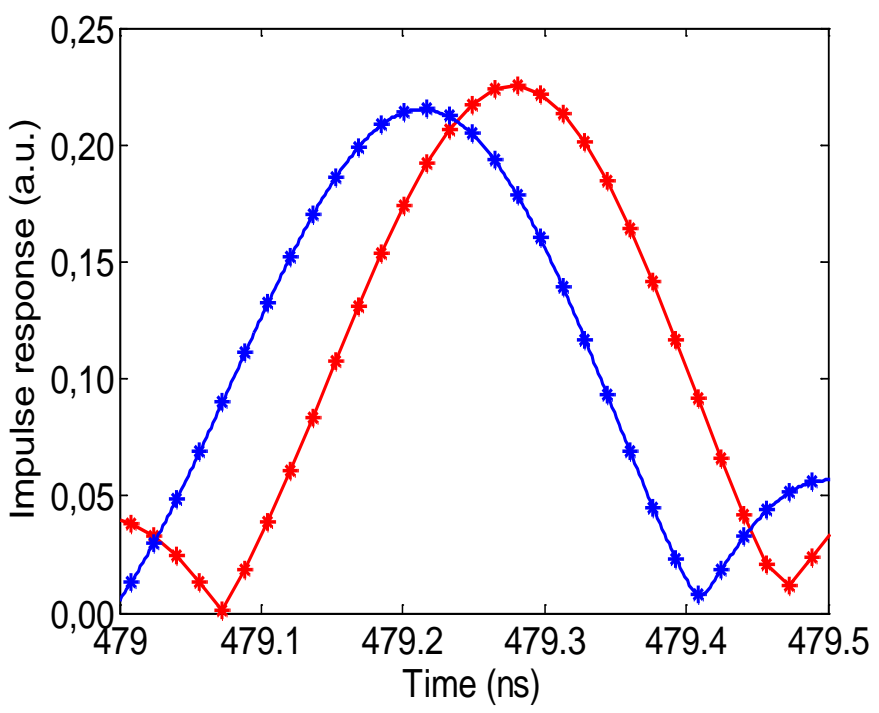

Fig. 8. Peaks in the system's impulse response generated by the FBG whose Bragg wavelength is $1540.6 \mathrm{~nm}$ with different impulse response resolution or temporal separation (crosses $=16 \mathrm{ps}$, dots $=1.6 \mathrm{ps}$ ) when the FBG is at the reference temperature (blue) and after heating (red). The high-resolution traces in dots are depicted as continuous curves. The IFT is computed by zeropadding the frequency response to one million points (dots) or 100000 points (crosses).

Comparison of Figs. 8 and 6 shows that the peak width has been enlarged by a factor of four due to the decrease in measurement bandwidth. Despite this fact, when the $5-\mathrm{GHz}$ VNA data is zero-padded up to one million points, we obtained the same value of the difference ( $8 \mathrm{pm})$ as with the original 32000 points, $20-\mathrm{GHz}$ trace in Fig. 6 . This is because the resolution in the peak determination depends on the sampling step $\Delta t^{\prime}$ and not to the peak's width. On the other hand, the Bragg wavelength shift measured after zero-padding up to only 100000 points is $0.5546 \mathrm{~nm}$, which represents a difference of $16.5 \mathrm{pm}$ with the measured trace with the OSA. This increment is due to the tenfold increase in sampling step. It is thus apparent that zero padding is a powerful tool that allows not only for a reduction of the measurement bandwidth, but also for reaching compromises between resolution and processing time through the decrease in the dimensionality of the zero-padded signal.

\section{CONCLUSIONS}

In this paper, a wavelength to radio-frequency delay mapping interrogation method of FBGs in cascade for its use as quasi- distributed temperature/strain sensors has been proposed and experimentally demonstrated. The principle uses Microwave Photonics techniques to map wavelength shifts into group delay shifts in the impulse response of a fiber optics link. The measurement is performed in the frequency domain by a VNA, and then the response translated to the time domain by numerical computation of the IFT. The scale for wavelength to delay conversion is determined by the total link's dispersion. The technique has been experimentally investigated in a cascade of seven FBGs with unequal reflected power and Bragg wavelength separation.

The resolution in the measurement of the Bragg wavelength is $14 \mathrm{pm}$, and is determined by the total dispersion used, the zero padding length of the raw VNA data, and its RF bandwidth. In principle, this resolution could be improved by enlarging the zero padding length up to a certain limit without requiring corrections of the linear relationship (3) (limit of 4 $\mathrm{pm}$ for a $1 \mathrm{~nm}$ of Bragg wavelength shift as stated in section II). Below this limit, an increase of resolution, a larger Bragg wavelength shift or both would require to take into account higher-order dispersion corrections to (3), and evaluate limitations due to overlapping of the impulse response peaks in (2). Besides, we have shown that, for a given resolution, the total RF measurement bandwidth required to implement the technique is scalable. Despite the increase in the peak's width, feasible wavelength shifts have been measured when the RF bandwidth is reduced from 20 to $5 \mathrm{GHz}$, thus avoiding the use of broadband microwave components. The method also shows an additional compromise between IFT processing time and resolution, through the length of the zero-padded signals. Finally, for a given processing effort, i.e., for a given temporal resolution in the impulse response $\Delta t^{\prime}$, the resolution can also be improved simply by enlarging the total dispersion in (3).

Although the cost of the equipment used, mainly the VNA, is important, its operational bandwidth could be reduced (for 20 to $5 \mathrm{GHz}$ ), or it could even be directly replaced by a tunable electrical oscillator and a dedicated digital signal processor to perform the same kind of measurements. Besides the flexibility, as exemplified in the above tradeoffs, the advantage over conventional approaches is that our proposed scheme does not require a tunable laser or an optical tunable receiver. To conclude, we point out that the same principle of operation can be used with cascaded FBG sensors where the gratings have the same Bragg wavelength and/or with a constant spectral separation between adjacent gratings.

\section{REFERENCES}

[1] K. Grattan and T. Sun, "Fiber optic sensor technology: an overview," Sens. Actuators A Phys., vol. 82, pp. 40-61, May, 2000.

[2] A.D. Kersey, "A review of recent developments in fiber optics sensor technology," Opt. Fiber Technol, vol. 2, pp. 291-317, Feb. 1996

[3] J. Hervás Peralta, A. Ricchiuti, D. Barrera, S. Sales, L. Thévenaz, and J. Capmany, "Weak fiber Bragg grating cascade sensor interrogation using microwave photonic filtering techniques," in Advanced Photonics, OSA Technical Digest (online) (Optical Society of America, 2014), paper SeW1C.2. 
[4] L. Thévenaz, S. Chin , J. Sancho, and S. Sales, “Novel technique for distributed fibre sensing based on faint long gratings (FLOGs),” Proc. SPIE, vol. 9157, 23rd International Conference on Optical Fibre Sensors, 91576W (June 2, 2014). [5] J. Huang, X. Lan, M. Luo, and H. Xiao, "Spatially continuous distributed fiber optic sensing using optical carrier based microwave interferometry," Opt. Express, vol. 22, pp. 18757-18769, 2014.

[6] A.L Ricchiuti, D. Barrera, S. Sales, L. Thévenaz, and J. Capmany, "Long fiber Bragg grating sensor interrogation using discrete-time microwave photonic filtering techniques," Opt. Express, vol. 21, pp. 28175-28181, Nov. 2013.

[7] M. Tateda and T. Horiguchi, "Advances in optical timedomain reflectometry," J. Lightw. Technol., vol. 7, no. 8, pp. 1217-1224, Aug. 1989.

[8] K. Yuksel, et al. "Optical frequency domain reflectometry: A review," presented at 11th International Conference on Transparent Optical Networks, 2009.

[9] J. Proakis and D. Manolakis, Digital Signal Processing, 4th. ed. Prentice-Hall, Upper Saddle River, NJ, 2006. 